\title{
2.16 MEASUREMENT OF THE CIRCULAR POLARIZATION OF PULSAR NP 0532
}

\author{
J. R. P. ANGEL* \\ Columbia Astrophysics Laboratory, Columbia University, New York, N.Y., U.S.A. \\ D. HEGYI** \\ NASA Goddard Institute for Space Studies N.Y,, New York, U.S.A. \\ J. D. LANDSTREET ${ }^{\dagger}$ \\ Department of Astronomy, Columbia University, New York, N.Y. U.S.A.
}

\begin{abstract}
The polarizer is briefly described. The polarization of the whole pulse is not significantly different from zero $(-0.15 \pm 0.70$ per cent). Polarization measurements of the leading edge and trailing edge of the main pulse are also given.
\end{abstract}

\section{Observations and Instrumentation}

We present below the results of measurement of the optical circular polarization of the pulsar NP 0532 . The observations were made on 11/12 February 1970 with the 82-in. telescope of McDonald Observatory.

The instrument used was the Cassegrain photoelectric polarimeter described by Angel and Landstreet (1970) and designed specifically for measuring circular polarization, together with a data handling system assembled for the occasion. The basic polarimeter consists of a KD*P electro-optic crystal (a Pockels cell) used as a reversible quarterwave plate, followed by a Wollaston prism which directs the light into two Channeltron photomultipliers. A periodically reversing square wave voltage is applied to the Pockels cell which causes one photomultiplier to measure alternatively the intensity of right and left circularly polarized light. The polarization of a star can thus be determined from the output of either photomultiplier alone.

The Pockels cell was set for quarter-wave retardation at about $5000 \AA$. The 1-mm S-20 photocathodes of the Channeltrons were unfiltered, but the dispersion of the beam by the Wollaston prism limits the light falling on the photocathodes to approximately the 4000-7000 $\AA$ range. The error in retardation in the Pockels cell for wavelengths other than $5000 \AA$ lowers the sensitivity of the system to circular polarization only a few percent.

The output signals from the photomultipliers were fed into two Northern 512 channel analyzers, one for each photomultiplier. The analyzers were used in a multiscaling mode; the channel address was advanced by a signal from a General Radio frequency synthesizer which was set to scan through the 511 data channels in exactly two pulsar periods. The voltage on the Pockels cell was reversed at the first and at

* Alfred P. Sloan Research Fellow.

** Present address: Department of Astronomy, Boston University, Boston, U.S.A.

$\dagger$ Present address: Department of Astronomy, University of Western Ontario, London, Canada. 
the 257th channels. Each multichannel analyzer thus recorded a full pulsar period in one circular polarization followed by a full period in the other polarization. It was not necessary for our purposes to go to great lengths to insure exact registration of the superposed data, but we may estimate from the data that even during the longest run (40 minutes) the phase error was no more than 2 or 3 channels, or less than $2 \%$.

Because this is a two-channel instrument, with measurements of both handednesses of circular polarization being done essentially simultaneously and through the same optical and electronic train, the systematic zero-point errors are extremely low (less than $0.05 \%$ ) and the measurement is not adversely affected by variations in transparency or background light. Our accuracy is thus limited mainly by statistical fluctuations in the signal and in the background light, which was primarily moonlight and whose amplitude equalled the peak height of the pulse.

The system was checked periodically by putting a circular polarizer (a piece of polaroid followed by a quarter-wave plate) in the beam before the Pockels cell. We also measured the circular polarization of comparison stars believed to be unpolarized. With the circular polarizer in the beam, we generally measure a polarization of more than $90 \%$, while the measured circular polarization of an unpolarized star is always less than $0.05 \%$.

TABLE I

Polarization of primary pulse of NP 0532

\begin{tabular}{ll}
\hline Component of pulse & $\begin{array}{l}\text { Measured polarization } \\
(\%)\end{array}$ \\
\hline Leading edge & $-0.19 \pm 0.89$ \\
Trailing edge & $-0.09 \pm 1.10$ \\
Whole pulse & $-0.15 \pm 0.70$ \\
Leading minus trailing edge & $-0.08 \pm 0.70$ \\
\hline
\end{tabular}

\section{Analysis and Results}

The fractional circular polarization was determined for the leading and trailing edges of the pulse separately by the following formula. Let the total number of counts in the leading edge of the pulse (which was taken to be the 15 channels $(1.9 \mathrm{msec}$ ) before the peak) be $N(l, R)$ and $N(l, L)$ in right and left circularly polarized light, and $N(b, R)$ and $N(b, L)$ be the corresponding numbers of counts of background light as given by the same number of channels, centered about $6 \mathrm{msec}$ before the main pulse. Then the fractional polarization is

$$
V(l)=\frac{[N(l, R)-N(b, R)]-[N(l, L)-N(b, L)]}{[N(l, R)-N(b, R)]+[N(l, L)-N(b, L)]}
$$

with a standard deviation given by

$$
\sigma(l)=\frac{\sqrt{N(l, R)+N(b, R)+N(l, L)+N(b, L)}}{[N(l, R)-N(b, R)]+[N(l, L)-N(b, L)]}
$$


Similar expressions apply for the trailing edge of the pulse, which is taken to be the 10 channels following the peak.

The resulting circular polarization from all runs summed together is shown in Table 1 for the leading and trailing edges of the primary pulse separately, for the whole pulse, and for the leading minus the trailing edge (this would be non-zero if the circular polarization changed sign at the peak of the pulse). It is seen that the circular polarization is zero to within the errors of measurement. This is in agreement with the previous results of Wampler et al. (1969), who find $V=3.9 \pm 4.9 \%$, and with the results of Cocke et al. (1969), who measure $V=2 \pm 1 \%$. The background nebula light is also found to be unpolarized.

This measurement was greatly reduced in accuracy by the fact that we made our observations on a moonlit night. We intend to repeat the measurement in the fall and expect to be able to reach a limit of no more than $0.1 \%$ uncertainty.

\section{Acknowledgements}

We are grateful to the Director of McDonald Observatory for the hospitality of the Observatory, and to Dr. Brian Warner and Dr. Patrick Thaddeus for their help and cooperation. This work was supported in part by the National Aeronautics and Space Administration, the Air Force Office of Scientific Research, and the Research Corporation. It is Columbia Astrophysics Laboratory Contribution No. 27.

\section{References}

Angel, J. R. P. and Landstreet, J. D.: 1970, Astrophys. J. Letters 160, L147.

Cocke, W. J., Disney, M. W., and Gehrels, T.: 1969, Nature 223, 576.

Wampler, E. J., Scargle, J. D., and Miller, J. S.: 1969, Astrophys. J. Letters 157, L1. 